\title{
A FISH-based method for assessment of HER-2 amplification status in breast cancer circulating tumor cells following CellSearch isolation
}

This article was published in the following Dove Press journal:

OncoTargets and Therapy

16 November 2016

Number of times this article has been viewed

\section{Henrik Frithiof' \\ Kristina Aaltonen' \\ Lisa Rydén ${ }^{2,3}$}

'Division of Oncology and Pathology, ${ }^{2}$ Division of Surgery, Department of Clinical Sciences Lund, Lund University, Lund, ${ }^{3}$ Department of Surgery, Skåne University Hospital, Malmö, Sweden
Correspondence: Lisa Rydén Division of Surgery, Department of Clinical Sciences Lund, Lund University, Medicon Village, SE-223 8I Lund, Sweden Tel +46 46 I 7624 I

Fax +46 $46 I 72335$

Email lisa.ryden@med.lu.se
Introduction: Amplification of the HER-2/neu (HER-2) proto-oncogene occurs in 10\%-15\% of primary breast cancer, leading to an activated HER-2 receptor, augmenting growth of cancer cells. Tumor classification is determined in primary tumor tissue and metastatic biopsies. However, malignant cells tend to alter their phenotype during disease progression. Circulating tumor cell (CTC) analysis may serve as an alternative to repeated biopsies. The Food and Drug Administration-approved CellSearch system allows determination of the HER-2 protein, but not of the HER-2 gene. The aim of this study was to optimize a fluorescence in situ hybridization (FISH)-based method to quantitatively determine HER-2 amplification in breast cancer CTCs following CellSearch-based isolation and verify the method in patient samples.

Methods: Using healthy donor blood spiked with human epidermal growth factor receptor 2 (HER-2)-positive breast cancer cell lines, SKBr-3 and BT-474, and a corresponding negative control (the HER-2-negative MCF-7 cell line), an in vitro CTC model system was designed. Following isolation in the CellSearch system, CTC samples were further enriched and fixed on microscope slides. Immunocytochemical staining with cytokeratin and 4',6-diamidino-2'phenylindole dihydrochloride identified CTCs under a fluorescence microscope. A FISH-based procedure was optimized by applying the HER2 IQFISH pharmDx assay for assessment of HER-2 amplification status in breast cancer CTCs.

Results: A method for defining the presence of HER-2 amplification in single breast cancer CTCs after CellSearch isolation was established using cell lines as positive and negative controls. The method was validated in blood from breast cancer patients showing that one out of six patients acquired CTC HER-2 amplification during treatment against metastatic disease.

Conclusion: HER-2 amplification status of CTCs can be determined following CellSearch isolation and further enrichment. FISH is superior to protein assessment of HER-2 status in predicting response to HER-2-targeted immunotherapy in breast cancer patients. This assay has the potential of identifying patients with a shift in HER-2 status who may benefit from treatment adjustments.

Keywords: neoplastic cells, circulating, breast neoplasms, human epidermal growth factor receptor 2 , in situ hybridization, fluorescence, immunomagnetic separation

\section{Introduction}

The human epidermal growth factor receptor 2 (HER-2) is a tyrosine kinase coupled receptor with a corresponding gene located on the long arm of chromosome 17q12-q21. HER-2/neu (HER-2) proto-oncogene amplification and the resultant protein overexpression are present in $\sim 10 \%-15 \%$ of primary invasive breast cancers, surmising an overall more aggressive cancer phenotype, essentially imparting a worse prognosis and 
short survival. ${ }^{1}$ HER-2-positive tumors show an increased inclination toward developing resistance to chemotherapy, while also being more common in younger patients. ${ }^{2,3}$ This subgroup of patients, as identified by immunohistochemistry (IHC) and fluorescence in situ hybridization (FISH) analysis of the primary tumor, benefit from immunotherapy with a monoclonal antibody trastuzumab (Herceptin ${ }^{\circledR}$ ), which specifically targets the extracellular domain of HER-2. Treatment leads to a significant increase in patient survival when used concomitantly with chemotherapy in the adjuvant setting. ${ }^{4-6}$

HER-2 classification is largely based on analysis of the primary tumor tissue. However, malignant cells tend to alter their phenotype during the course of disease progression, thus the tumor profile as defined by biopsies resected at one stage will not necessarily outline future phenotypical shifts. This is emphasized by previous findings of discordance rates of $7 \%-37 \%$, comparing HER-2 status in primary tumors with corresponding metastases. $^{7-13}$ In this context, analysis of circulating tumor cells (CTCs) represents an accessible alternative to iterative biopsies. CTCs are malignant cells that detach from the primary tumor or metastases and are detected in the blood stream of patients with both early and late stage carcinomas. Studies have suggested that both HER-2 activation and deactivation during breast cancer progression is possible, as indicated by isolation of HER-2-positive CTCs in patients initially classified as HER-2 negative and vice versa, based on primary tumor protein expression and/or HER-2 amplification. ${ }^{14-16}$ These observations argue that the true number of patients eligible for HER-2-targeted immunotherapy is in fact higher. Additionally, continuous evaluation of HER-2 status in CTCs might be a well-suited, clinically valid surrogate marker in the setting of minimal residual disease.

During the last decade, the enumeration of CTCs has been acknowledged as an important prognostic tool in predicting progression-free and overall survival in metastatic breast cancer (MBC), as well as a prognostic factor for early breast cancer in terms of recurrence-free and overall survival. ${ }^{17,18}$ However, studies of predictive strength related to the number of CTCs in the subset of patients with HER-2-positive disease treated with targeted therapy are not quite as unanimous, with slightly deviating results depending on selected numerical CTC cutoff values. ${ }^{19,20}$ This is largely contributed to the propensity of anti-HER-2 therapies in substantially decreasing the CTC quantity and improving patient prognosis.

The gold standard technology for CTC isolation is the Food and Drug Administration (FDA)-approved CellSearch technology (Janssen Diagnostics, Raritan, NJ, USA), which relies on epithelial cell adhesion molecule (EpCAM)-based immunomagnetic separation and has previously been described in detail elsewhere. ${ }^{21}$

Our group has recently published a consistent immunofluorescence (IF)-based method for simultaneous HER-2 and estrogen receptor (ER)- $\alpha$ protein analysis in $\mathrm{MBC}$ patient CTCs, based on the CellSearch platform. ${ }^{22}$ While IHC is extensively used in the clinic for initial HER-2 assessment, FISH is considered the benchmark test within this particular field. Clinically, FISH is superior to IHC in predicting response to HER-2-targeted immunotherapy in breast cancer patients. ${ }^{23}$ HER-2 protein assessment can be performed using the CellSearch system, whereas no known protocol is established for FISH-based analysis with this platform. Previous data on HER-2 status by FISH from primary tumors and CTCs are not derived from the CellSearch system but from a non-FDA-approved method which is not commercially available. ${ }^{14,15}$

We sought to extend the possibility to determine HER-2 status in CTCs captured with the widely used, FDA-approved CellSearch system by developing a protocol for assessment of HER-2 amplification. The aim of this study was to optimize a FISH-based method to quantitatively determine HER-2 amplification in CTCs from patients with MBC following CellSearch-based isolation.

\section{Methods \\ CellSearch}

The CellSearch system is a semiautomated assay based on immunomagnetic separation involving positive selection of CTCs by means of magnetic ferrofluid-associated antiEpCAM antibodies. ${ }^{24}$ This technique defines CTCs as cells with an intracellular nucleus, a size of at least $4 \times 4 \mu \mathrm{m}^{2}$, and positive for cell markers EpCAM, cytokeratin (CK) 8, 18 , or 19 , while negative for the leukocyte-specific surface protein, cluster of differentiation 45 (CD45). ${ }^{21}$

\section{Patients}

Ethical permission for the CTC-MBC study was obtained from Lund University Ethical Board (EPN 2010/135) and all patients gave a written informed consent.

All patients had MBC and had been included before start of first-line systemic therapy in the ongoing CTC-MBC trial (NCT01322893) at Lund University, Sweden. In this trial, serial sampling is performed prior to, and after disease progression, enabling evaluation of biomarker changes in CTCs related to progressive disease. Patient selection 
in the present study was based on the premise of having detectable CTCs with CellSearch analysis, either at baseline or 3-12 months from baseline.

\section{Cell lines and in vitro model design}

HER-2 amplification in CTCs using CellSearch was calibrated through peripheral healthy donor blood-spiking experiments with EpCAM-positive breast cancer cell lines MCF-7, SKBr-3, and BT-474. SKBr-3 and BT-474 are both HER-2 positive, while MCF-7 is HER-2 negative and was therefore selected as a negative control. Short tandem repeat-authenticated cell lines were obtained from the American Type Culture Collection (ATCC/LGC Standards GmbH, Wesel, Germany). These cells were grown in a $5.0 \% \mathrm{CO}_{2}$ incubator under ultraviolet light at $37^{\circ} \mathrm{C}$ in culture vessels containing $5 \mathrm{~mL}$ media. MCF-7 cells were grown in $5 \mathrm{~mL}$ minimum essential medium with Earle's balanced salt solution (HyClone Laboratories, Inc., Logan, UT, USA) medium supplemented with $1 \%$ sodium pyruvate, $1 \%$ nonessential amino acids, $10 \%$ fetal bovine serum (FBS), and 1\% penicillin-streptomycin mixture (Pen-Strep). SKBr-3 and BT-474 cells were cultured in Roswell Park Memorial Institute 1640 (HyClone Laboratories, Inc.) supplemented with 10\% FBS, $10 \mathrm{mM}$ 4-(2-hydroxyethyl)-1-piperazineethanesulfonic acid, and 1\% Pen-Strep. Harvesting of cells was performed at $80 \%-90 \%$ culture confluence after 5-10 min trypsinization.

Peripheral blood samples were processed within 24 hours from withdrawal, and spiking of cells occurred just prior to CellSearch analysis. Approximately, $7.5 \mathrm{~mL}$ of healthy donor blood was spiked with cell dilutions of $\sim 2,000$ cells and the sample was subsequently processed with the CellSearch Profile kit according to the manufacturers' protocol. ${ }^{24}$

\section{CTC-DropMount}

The enriched samples were mounted on individual glass slides using an in-house developed procedure, denoted CTC-DropMount. ${ }^{22}$ Briefly, the CTC-containing solution $(\sim 900 \mu \mathrm{L})$ was transferred to a $1.5 \mathrm{~mL}$ Eppendorf tube and placed in a magnetic tray. Following 10 min incubation, the nonadherent solvent was removed. The cells were resuspended in $10 \mu \mathrm{L}$ of $1 \times$ phosphate buffer saline and mounted on superfrost slides (ThermoScientific, Germany) which were incubated at $37^{\circ} \mathrm{C}$ for $30 \mathrm{~min}$. Fixation was accomplished by immersing the slides in $100 \%$ methanol for $5 \mathrm{~min}$. The samples were stored at $-20^{\circ} \mathrm{C}$. Using a triple IF staining protocol tailored for CTCs, the samples were assessed for expression of CK/phycoerythrin (PE), ER- $\alpha$ /AlexaFluor488, CD45/ allophycocyanin (APC), and 4',6-diamidino-2'-phenylindole
(DAPI). Each slide was individually assessed and scanned with a fluorescence microscope; areas containing CTCs were outlined in the image to assure subsequent location and identification of CTCs following FISH staining. That is, cells positive for the expression of CK, DAPI, and simultaneously negative for the leukocyte-specific marker, CD45. This step is imperative because FISH treatment obliterates any remaining IF stain in the sample. Following IF staining, the samples were stored at $+4^{\circ} \mathrm{C}$. The recovery rate of CTC-DropMount has previously been found to be $87 \%$ on average, using CellSearch Profile analysis from whole blood. The immunostaining procedure for assessment of CK, CD45, ER- $\alpha$, and HER-2 was described in detail in an earlier study. ${ }^{22}$

\section{FISH protocol for HER-2 amplification in breast cancer CTCs}

Pretreatment, denaturation, hybridization, and staining of slides were adjusted based on the FDA-approved HER2 IQFISH pharmDx assay, and the IQISH hybridization buffer chemistry provided by Dako (Dako Denmark A/S, Glostrup, Denmark).

The optimized procedure protocol for CTCs is detailed in Table 1. Cell fixation was evaluated in pure methanol (\#I659409, Merck KGaA, Darmstadt, Germany), 1\% formaldehyde, and a mixture of methanol and acetate (3:1). Cell permeabilization was achieved using Dako Target Retrieval solution containing Tris/ethylenediaminetetraacetic acid buffer solution pH 9.0 and detergent (\#20020172, Dako Denmark, A/S); incubation times assessed were 5-15 min at $37^{\circ} \mathrm{C}$ or $95^{\circ} \mathrm{C}$. Incubation times assessed for the pepsin treatment were $2-15 \mathrm{~min}$, at either room temperature or $37^{\circ} \mathrm{C}$. Chromosome enumerator probe 17 (CEP-17) labeled with fluorescein-conjugated peptide nucleic acids, and the HER-2-specific probe conjugated with TexasRed were provided by Dako. Slides were counterstained and prepared with mounting medium containing DAPI.

HER-2 amplification analysis was performed at $60 \times$ magnification with an Olympus BX63 microscope (Olympus Optical CO., Hamburg, Germany) equipped with a digital DP80 camera and single pass filters for DAPI, GFP/Alexa488, and PE/TexasRed. HER-2-amplified CTCs were defined as having a $H E R-2 / \mathrm{CEP}-17$ ratio of $>2$, or with average $H E R-2$ copy number $\geq 6$ signals per cell, according to current breast cancer pathology recommendations. ${ }^{25}$

\section{In vivo validation}

Patient blood samples were investigated for clinical validation of the technique. Using the CellSearch Profile kit, 
Table I Optimized FISH protocol for assessment of HER-2 amplification status in breast cancer CTCs

\begin{tabular}{|c|c|c|c|c|}
\hline Step & Reagent & Concentration & Interval/temperature & Manufacturer/batch \\
\hline \multicolumn{5}{|l|}{ Pretreatment } \\
\hline Cell fixation ${ }^{\mathrm{a}}$ & Methanol & $\mathrm{I}: \mathrm{I}$ & $5 \mathrm{~min}$ at $\mathrm{RT}$ & $\begin{array}{l}\text { Merck KGaA (Darmstadt, Germany), } \\
\# 1659409\end{array}$ \\
\hline Cell permeabilization $^{\mathrm{a}}$ & $\begin{array}{l}\text { Dako pretreatment } \\
\text { solution } 20 \times\end{array}$ & $\mathrm{I}: 20$ & $10 \min$ at $95^{\circ} \mathrm{C}$ & $\begin{array}{l}\text { Dako Denmark (Glostrup, Denmark), } \\
\text { A/S, \#20020I72 }\end{array}$ \\
\hline Pepsin incubation ${ }^{\mathrm{a}}$ & Pepsin & $\mathrm{I}: \mathrm{I}$ & $2 \mathrm{~min}$ at $37^{\circ} \mathrm{C}$ & Dako Denmark, A/S, \#20020172 \\
\hline Dehydration & $\begin{array}{l}\text { Analytical grade } \\
\text { ethanol, } 99.5 \%\end{array}$ & $70 \% ; 80 \% ; 95 \%$ & $3 \times 2 \mathrm{~min}$ at $\mathrm{RT}$ & $\begin{array}{l}\text { Solveco (Rosersberg, Sweden), } \\
\text { \#6088756 }\end{array}$ \\
\hline CEP-I7/HER-2 probe & HER2/CEP-I7 & $\mathrm{I}: \mathrm{I}$ & & Dako Denmark, A/S, \#200I9055 \\
\hline $\begin{array}{l}\text { application } \\
\text { Add coverslip and sealant }\end{array}$ & $\begin{array}{l}\text { IQISH probe mix } \\
\text { Coverslip sealant }\end{array}$ & & & \\
\hline \multicolumn{5}{|l|}{ Denaturation and } \\
\hline \multicolumn{5}{|l|}{ hybridization $^{\mathrm{b}}$} \\
\hline $\begin{array}{l}\text { Preheat hybridization } \\
\text { chamber }\end{array}$ & & & $66^{\circ} \mathrm{C}$ & \\
\hline Denaturation & & & $10 \mathrm{~min}$ at $66^{\circ} \mathrm{C}$ & \\
\hline Hybridization & & & $90 \mathrm{~min}$ at $45^{\circ} \mathrm{C}$ & \\
\hline \multicolumn{4}{|l|}{ Stringent wash } & \\
\hline Wash \#I & $\begin{array}{l}\text { Dako stringent } \\
\text { wash buffer } 20 x\end{array}$ & $\mathrm{I}: 20$ & I $\min$ at $\mathrm{RT}$ & Dako Denmark, A/S, \#20020I72 \\
\hline Wash \#2 ${ }^{\mathrm{a}}$ & $\begin{array}{l}\text { Dako stringent } \\
\text { wash buffer } 20 x\end{array}$ & $\mathrm{I}: 20$ & $10 \min$ at $63^{\circ} \mathrm{C}$ & Dako Denmark, A/S, \#20020I72 \\
\hline Dehydration & $\begin{array}{l}\text { Analytical grade } \\
\text { ethanol, } 99.5 \%\end{array}$ & $70 \% ; 80 \% ; 95 \%$ & $3 \times 2$ min at $R T$ & Solveco, \#6088756 \\
\hline \multicolumn{5}{|l|}{ Mounting and reading } \\
\hline Nuclear counterstaining & $\begin{array}{l}\text { Fluorescence } \\
\text { mounting medium, } \\
\text { DAPI }\end{array}$ & $\mathrm{I}: \mathrm{I}$ & & Dako Denmark, A/S, \#200I9055 \\
\hline Add coverslip and sealant & Coverslip sealant & & & \\
\hline
\end{tabular}

Notes: aWashing with iced phosphate buffer saline $10.0 \%(\mathrm{v} / \mathrm{v}), 3 \times 3 \mathrm{~min}$ following this step. ${ }^{\mathrm{b}}$ This step could also be accomplished using an automatic hybridizer, described here is the manual procedure.

Abbreviations: CEP-I7, chromosome enumerator probe 17; CTC, circulating tumor cell; DAPI, 4',6-diamidino-2'-phenylindole; FISH, fluorescence in situ hybridization; HER-2, human epidermal growth factor receptor 2; A/S, stock-based company; RT, room temperature.

altogether 8 clinical samples of $7.5 \mathrm{~mL}$ peripheral blood from six individual patients were analyzed. Each sample was initially stained by IF for initial assessment of CK, CD45, and ER- $\alpha$ status and scanned with the microscope, as described before. FISH analysis was processed in conjunction with positive and negative controls, represented by SKBr-3 or BT-474 (HER2 ${ }^{+}$) and MCF-7 (HER2-) cell lines, respectively.

\section{Results}

CTCs were defined according to CellSearch criteria, as nucleated (DAPI+), epithelial (positive for CK 8, 18, or 19), and CD45 negative. The protocol for assessment of HER-2 amplification status in CTCs by FISH was verified in breast cancer cell line cells with known HER-2 status (Table 1). Turnover time for FISH staining was $\sim 4$ hours. Fixation by pure methanol was deemed superior compared to $1 \%$ formaldehyde or methanol/acetate (3:1) mixture by visual inspection, showing consistently higher preservation of cellular morphology. The procedure was considered optimized in regards to pretreatment, denaturation, and hybridization, when both SKBr-3 and BT-474 were consistently stained HER-2 amplified, and MCF-7 was non-amplified, while maintaining nuclear and cytoplasmic integrity (Figure 1). Cell permeabilization proved most effective at the higher temperature $\left(95^{\circ} \mathrm{C}\right)$, with an incubation time of $10 \mathrm{~min}$. A shorter interval or lower temperature $\left(37^{\circ} \mathrm{C}\right)$ resulted in less technical reproducibility, with higher intercellular variation in HER-2 amplification status in the HER-2-positive cell lines. Pretreatment with pepsin showed similar results in cell line samples up to $5 \mathrm{~min}$, but patient samples proved too fragile for treatment $>2$ min with cellular distension and increased loss of nuclear membrane integrity beyond this point. A slight background staining was unavoidable in all samples, due to some remaining ferrofluid microparticles. HER-2/CEP-17 ratios in the HER-2-positive cell lines were consistent with previous research works; $\mathrm{SKBr}-3$ had 4- to 8-fold amplification, and BT-474 had 6- to 10-fold amplification. ${ }^{26}$ 

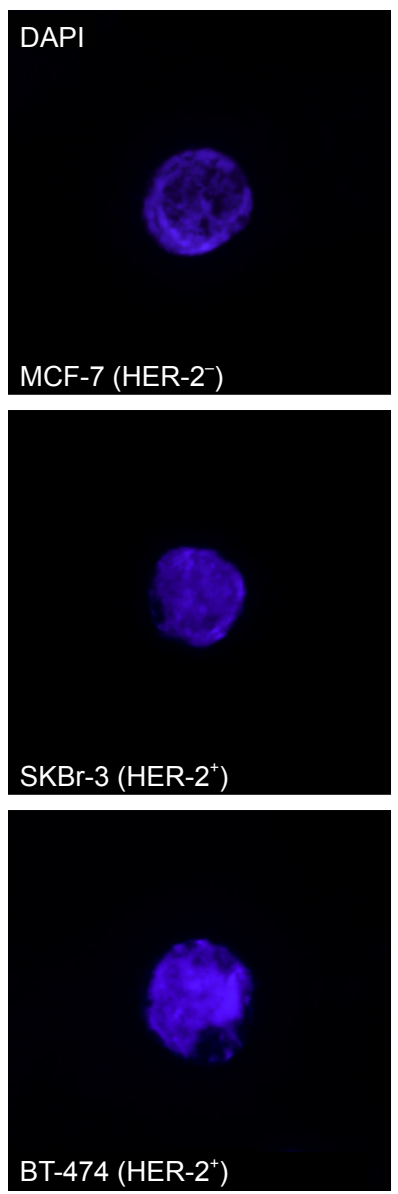
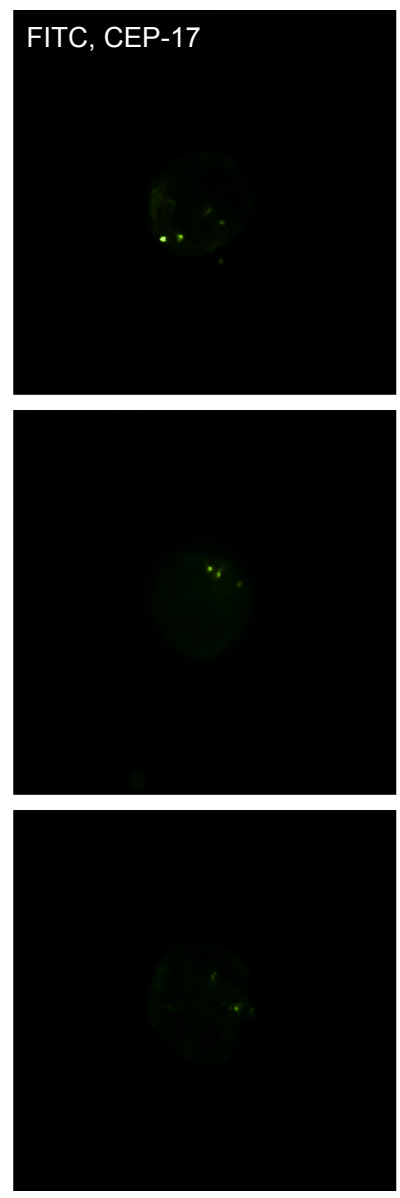
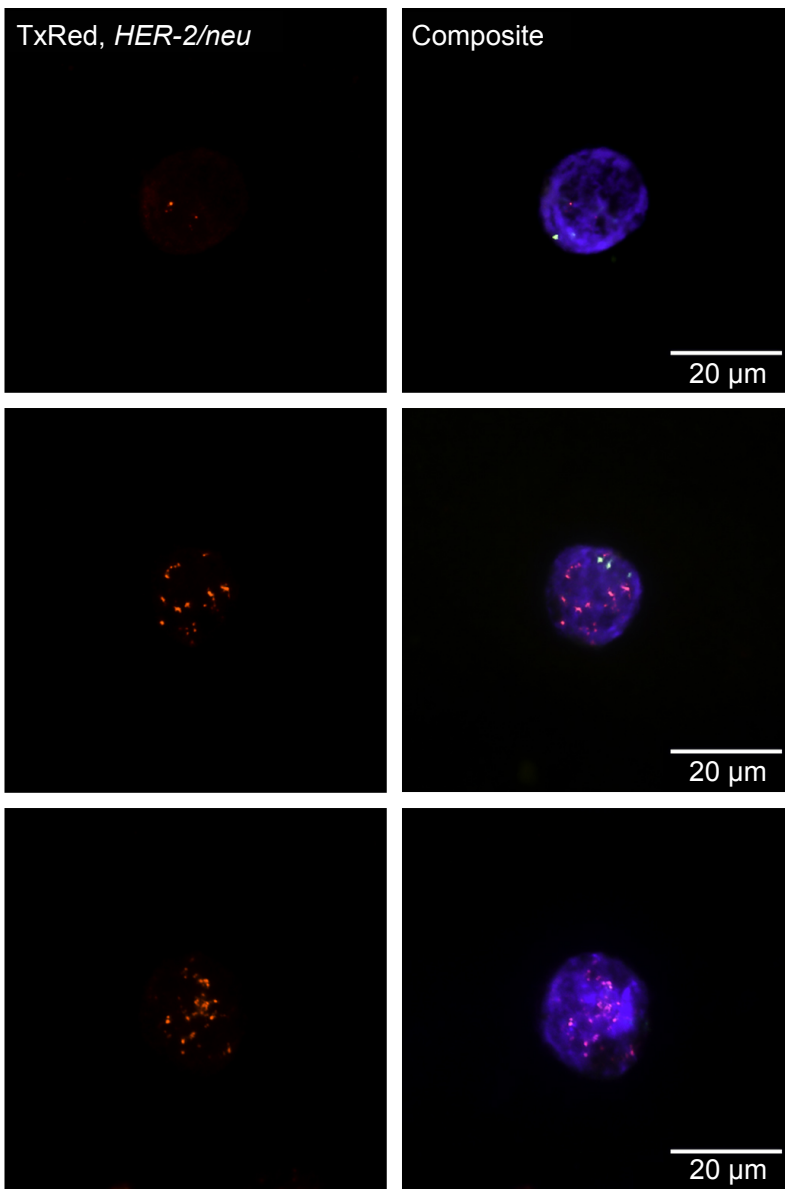

Figure I FISH HER-2 staining of MCF-7, SKBr-3, and BT-474 cell line cells.

Notes: Selective HER-2 amplification was demonstrated in SKBr-3 and BT-474 cells, in contrast to the non-amplified MCF-7 cells. The cell lines were used for staining optimization in a CTC in vitro model system. From left to right: DAPI counterstain (fluorescent blue), CEP-I7 stained with fluorescein isothiocyanate (green), HER-2 stained with TexasRed (red), and a composite of all channels.

Abbreviations: CEP-17, chromosome enumerator probe 17; CTC, circulating tumor cell; DAPI, 4',6-diamidino-2'-phenylindole; FISH, fluorescence in situ hybridization; HER-2, human epidermal growth factor receptor 2.

The method was assessed in eight clinical samples (Table 2) for patient characteristics including phenotypes of primary tumor and metastasis, total number of CTCs detected, and results of FISH HER-2 analysis. All baseline samples were $H E R-2$ negative, whereas one patient presented with HER-2-amplified CTCs during treatment for MBC. Initially, this patient (no 3, Table 2) had no detectable CTCs with HER-2 amplification, however, at 3 months follow-up one

Table 2 Patient data for in vivo validation procedures

\begin{tabular}{|c|c|c|c|c|c|}
\hline Patient no & Time frame & $\begin{array}{l}\text { Primary tumor } \\
\text { phenotype }\end{array}$ & $\begin{array}{l}\text { Metastasis } \\
\text { phenotype }\end{array}$ & Number of CTCs ${ }^{a}$ & CTC FISH-HER-2 ${ }^{b}$ \\
\hline I & $\mathrm{BL}$ & $\mathrm{ER}^{+} / \mathrm{HER}-2^{-}$ & $\mathrm{ER}^{+} / \mathrm{HER}^{-2^{-}}$ & 263 & Non-amplified \\
\hline 2 & $B L$ & ER-/HER-2- & N/A & 5 & Non-amplified \\
\hline \multirow[t]{3}{*}{3} & $B L$ & $\mathrm{ER}^{+} / \mathrm{HER}^{-2^{-}}$ & $\mathrm{ER}^{+} / \mathrm{HER}-2^{-}$ & 124 & Non-amplified \\
\hline & 3 months from BL & & & 13 & Amplified \\
\hline & 6 months from BL & & & 8 & Amplified \\
\hline 4 & $\mathrm{BL}$ & $\mathrm{ER}^{+} / \mathrm{HER}-2^{-}$ & $\mathrm{ER}^{+} / \mathrm{HER}-2^{-}$ & 2,598 & Non-amplified \\
\hline 5 & 9 months from BL & $\mathrm{ER}^{+} / \mathrm{HER}-2^{-}$ & N/A & 120 & Non-amplified \\
\hline 6 & 12 months from BL & N/A & $\mathrm{ER}^{+} / \mathrm{HER}-2^{-}$ & 37 & Non-amplified \\
\hline
\end{tabular}

Notes: ${ }^{\mathrm{A} A s}$ defined by CellSearch, single samples assessed $0-12$ months from initiation of therapy against metastatic disease. ${ }^{\mathrm{b}} \mathrm{Criteria}$ for biomarker positivity was $\geq \mathrm{I}$ HER- $2^{+}$ CTC, that is, the HER-2/CEP-I7 ratio of $>2$, or with an average HER-2 copy number $\geq 6$ signals per cell.

Abbreviations: BL, baseline; CEP-17, chromosome enumerator probe 17; CTC, circulating tumor cell; ER, estrogen receptor; FISH, fluorescence in situ hybridization; HER-2, human epidermal growth factor receptor 2; N/A, not available. 

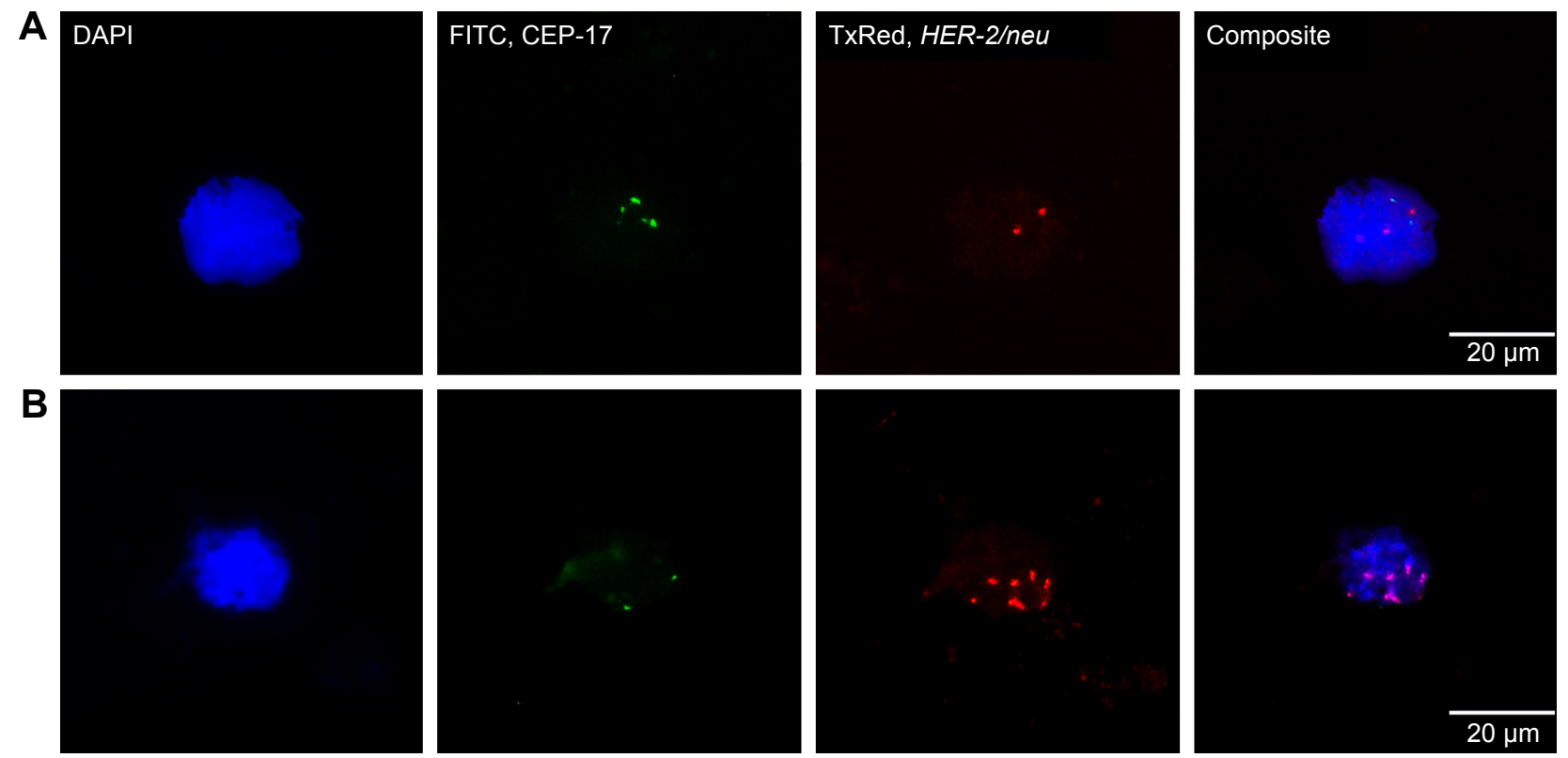

Figure 2 FISH HER-2 staining of metastatic breast cancer blood samples.

Notes: (A) Left to right: DAPI counterstain (fluorescent blue), CEP-I7 stained with fluorescein isothiocyanate (green), HER-2 stained with TexasRed (red), and a composite of all channels. Representative image of a patient's CTC without detectable HER-2 amplification. (B) Left to right: DAPI counterstain (fluorescent blue), CEP-I7 stained with fluorescein isothiocyanate (green), HER-2 stained with TexasRed (red), and a composite of all channels. Example of a HER-2-amplified CTC identified in a patient who presented with a HER-2-negative primary tumor and metastases (no 3 in Table 2).

Abbreviations: CEP-I7, chromosome enumerator probe 17; CTC, circulating tumor cell; DAPI, 4',6-diamidino-2'-phenylindole; FISH, fluorescence in situ hybridization; HER-2, human epidermal growth factor receptor 2 .

CTC showed apparent amplification with an HER-2/CEP-17 ratio of 4, and at 6 months follow-up one CTC was amplified with an HER-2 copy number of nine signals. Examples of HER-2-negative and HER-2-amplified patient CTCs are shown in Figure 2.

\section{Discussion}

Although a quantitative approach to CTCs has its confirmed merits in regards to patient prognosis, phenotyping, and in-depth genomic analysis of these rare cells remain a potential source of clinically vital information for selection of systemic therapy, representing a largely unexplored area of contemporary cancer cytopathology.

In the present study, we aimed to set up a method for assessment of HER-2 amplification status in order to further define CTCs in patients with MBC following isolation with the CellSearch technology. The procedure was initially calibrated using established breast cancer cell lines added to healthy donor peripheral blood, followed by in vivo validation in samples obtained from patients with MBC. Furthermore, this method has the advantage of combining assessment of total number of CTCs and morphological evaluation along with staining of $\mathrm{CK}, \mathrm{CD} 45$, and $\mathrm{ER} \alpha$, prior to FISH of HER-2 amplification, ${ }^{22}$ thereby ultimately extracting abundant clinically important information from a minute sample of $7.5 \mathrm{~mL}$ blood.
HER-2 is commonly considered the second most important prognostic and treatment predictive biomarker in breast cancer. Discordance in HER-2 expression when comparing primary tumor with metastases is frequently described in the literature, varying from $7 \%$ to $37 \% .^{7-13}$ This is often attributed to genuine changes in tumor biology: signifying clonal selection caused by systemic therapy, intratumoral heterogeneity, or differential shifts in expansion of breast cancer stem cells. ${ }^{27-29}$ Theoretically, an early cancer stem cell or an aggressive non-stem cell subclone may migrate to a distant site and establish a metastatic lesion with features distinctly separate from those of the primary tumor. Heterogeneity may also in part be explained by the inherent genetic instability in breast cancer cells. In contrast, Pusztai et al have previously advocated that common technical confounders, such as sampling errors due to tumors containing several foci with different receptor statuses, and inadequate accuracy and variable reproducibility of receptor analyses should also be taken into account when comparing primary tumors and secondary lesions or CTCs, in regards to either HER-2 or hormone receptor status. ${ }^{30}$ This underlines the importance of adhering to established guidelines for biomarker analysis also in metastatic tissues and CTCs.

In an exploratory study, Meng et al were among the first to determine HER-2 amplification in breast cancer CTCs using FISH after immunomagnetic separation and identification 
of CTCs under the microscope. They proposed that gene amplification can be acquired as cancer progresses, as 10 of 24 patients with an $H E R$-2-negative primary tumor acquired CTC HER-2 amplification during disease progression. ${ }^{14}$ Other studies using CellSearch in combination with integrated IF staining of the HER-2 protein supported these findings, reporting CTC HER-2 discordance rates of HER-2 protein expression ranging between $18 \%$ and $42 \%$ in patients with HER-2-negative primary tumors. ${ }^{16,31-33}$ However, no method for downstream HER-2 analysis by FISH after CTC isolation with the CellSearch system has been reported. Consequently, no data on HER-2 status by FISH comparing primary tumors and CTCs isolated by CellSearch have been presented. Swennenhuis et al utilized a modified CellSearch system, fixating CTCs from patients with metastatic prostate cancer within the magnetic cartridge following complete CellSearch analysis, then using FISH analysis for successful characterization of different chromosomal aberrancies, not including HER-2 amplification. ${ }^{34}$ However, this particular method was associated with high levels of cellular disintegration, mainly attributed to apoptosis of CTCs, which partly restricts its analytical validity.

The major drawback of using IHC as means of HER-2 determination in CTCs is unspecified accuracy; also, the cutoff values regarding staining intensity are less than obvious. In contrast, FISH has clearly defined criteria in what constitutes amplified versus non-amplified cells according to established guidelines. Acknowledging the fact that the sample size in the present study is small, and its intentions being primarily exploratory, the results nonetheless indicate a clinical potential of the method in identifying patients eligible for HER-2-targeted treatment based on CTC HER-2 status. One out of the six patients included in the in vivo validation procedures seemingly acquired HER-2 amplification during treatment against metastatic disease (Table 2). While initially being classified as CTC HER-2 negative at baseline, the following two sequential samples revealed CTCs with HER-2 amplification. The start of systemic treatment substantially reduced the number of CTCs in this patient, starting at 124 detected cells at baseline, followed by 13 and 8 CTCs detected at 3 and 6 months follow-up, respectively. Hypothetically, the additional selection pressure represented by a novel treatment regime may in turn have exposed and contributed to intratumoral conditions favoring clonal selection of $H E R$-2-amplified cells. In this patient, it is conceivable that the addition of HER-2-targeted therapy could aid in further suppression of the disease. All the remaining patients demonstrated CTCs without detectable HER-2 amplification.
Underlining the relevance of accurate HER-2 analysis in CTCs as well as the clinical potential of alterations in CTC HER-2 expression during disease development, several large-scale prospective interventional trials are currently investigating the predictive importance of such findings. ${ }^{35}$ The DETECT III trial (NCT016119111) is a two-arm phase III study assessing whether patients with HER-2-negative metastatic disease, but with $\geq 1$ HER-2-positive CTC as identified by IF, benefit from treatment with HER-2-targeted therapy with lapitinib. Based on the same premise, the CirCe T-DM1 trial (NCT01975142) explores the validity of HER-2-amplified CTCs, also taking into account the number of detected CTCs with amplification (either $1-4$ or $\geq 5$ ), in selecting MBC patients initially classified as HER-2 negative for combined trastuzumab emtansine (T-DM1) treatment. The method for combining CellSearch with FISH and the specific FISH protocol used in this study is not publically available. Treat CTC (NCT01548677) is another multicenter trial involving CTCs, focusing on patients with HER-2negative non-metastatic disease and $\geq 1$ detectable CTC, regardless of their HER-2 status, in peripheral blood. The rationales for this study are findings indicating that adjuvant treatment with trastuzumab may benefit patients irrespective of HER-2 amplification status in the primary tumor, applying CTCs as a surrogate marker for response to therapy. ${ }^{36,37}$ This trial uses detectable level of CTCs at 18 weeks from inclusion as a primary endpoint.

\section{Conclusion}

HER-2 amplification status of CTCs was possible to determine following CellSearch isolation and further enrichment in a minute blood sample applying the presented protocol, defining HER-2 amplification according to clinical guidelines. This assay has the potential of identifying patients with a shift in HER-2 status from primary tumors to CTCs isolated by the CellSearch system. These patients may in the future benefit from treatment adjustments if ongoing trials are able to show that treatment predictive information for HER-2 directed therapy can be obtained from HER-2 status on CTCs. The clinical utility of determining HER-2 status on CTCs following CellSearch isolation warrants confirmation in larger cohorts of patients with MBC with detailed information on treatment and follow-up.

\section{Acknowledgments}

This study was supported by the Swedish Cancer Foundation (CAN 2013/533), Swedish Research Council (2015-02516), the Gunnar Nilsson Cancer Foundation (2013/1224), the Mrs Berta Kamprad Foundation (2014/36), the Crafoord 
Foundation (2013/0563), Governmental funding of clinical research within the national health service (ALF) (2014/434901), the Skåne County council's research and development foundation (2014/452081), Swedish Breast Cancer Organization (BRO), and partly by Roche AB, Sweden.

\section{Author contributions}

HF: Performed and validated all steps in methodology and investigation. Validated and visualized the staining procedure. KA: Conceptualized the study; responsible for the technical development of the individual steps in methodology including validation and visualization of the staining procedure. LR: Designed, initiated, and conceptualized the study including study design. All authors took part in drafting the article or revising it critically for important intellectual content; gave final approval of the version to be published; and agree to be accountable for all aspects of the work.

\section{Disclosure}

The authors report no conflicts of interest in this work.

\section{References}

1. Slamon DJ, Clark GM, Wong SG, Levin WJ, Ullrich A, McGuire WL. Human breast cancer: correlation of relapse and survival with amplification of the HER-2/neu oncogene. Science. 1987;235(4785): 177-182.

2. Slamon D, Godolphin W, Jones L, et al. Studies of the HER-2/neu proto-oncogene in human breast and ovarian cancer. Science. 1989; 244(4905):707-712.

3. Gusterson BA, Gelber RD, Goldhirsch A, et al. Prognostic importance of c-erbB-2 expression in breast cancer. International (Ludwig) Breast Cancer Study Group. J Clin Oncol. 1992;10(7):1049-1056.

4. Baselga J. Herceptin alone or in combination with chemotherapy in the treatment of HER2-positive metastatic breast cancer: pivotal trials. Oncology. 2001;61 (Suppl 2):14-21.

5. Davoli A, Hocevar BA, Brown TL. Progression and treatment of HER2positive breast cancer. Cancer Chemother Pharmacol. 2010;65(4): 611-623.

6. Piccart-Gebhart MJ, Procter M, Leyland-Jones B, et al. Trastuzumab after adjuvant chemotherapy in HER2-positive breast cancer. $N$ Engl $J$ Med. 2005;353(16):1659-1672.

7. Chang HJ, Han SW, Oh DY, et al. Discordant human epidermal growth factor receptor 2 and hormone receptor status in primary and metastatic breast cancer and response to trastuzumab. Jpn J Clin Oncol. 2011;41(5):593-599.

8. Zidan J, Dashkovsky I, Stayerman C, Basher W, Cozacov C, Hadary A. Comparison of HER-2 overexpression in primary breast cancer and metastatic sites and its effect on biological targeting therapy of metastatic disease. Br J Cancer. 2005;93(5):552-556.

9. Wilking U, Karlsson E, Skoog L, et al. HER2 status in a populationderived breast cancer cohort: discordances during tumor progression. Breast Cancer Res Treat. 2011;125(2):553-561.

10. Lower EE, Glass E, Blau R, Harman S. HER-2/neu expression in primary and metastatic breast cancer. Breast Cancer Res Treat. 2009;113(2): 301-306.

11. Gancberg D, Di Leo A, Cardoso F, et al. Comparison of HER-2 status between primary breast cancer and corresponding distant metastatic sites. Ann Oncol. 2002;13(7):1036-1043.
12. Pectasides D, Gaglia A, Arapantoni-Dadioti P, et al. HER-2/neu status of primary breast cancer and corresponding metastatic sites in patients with advanced breast cancer treated with trastuzumab-based therapy. Anticancer Res. 2006;26(1B):647-653.

13. Niikura N, Liu J, Hayashi N, et al. Loss of human epidermal growth factor receptor 2 (HER2) expression in metastatic sites of HER2-overexpressing primary breast tumors. J Clin Oncol. 2012;30(6): 593-599.

14. Meng S, Tripathy D, Shete S, et al. HER-2 gene amplification can be acquired as breast cancer progresses. Proc Natl Acad Sci US A. 2004; 101(25):9393-9398.

15. Meng S, Tripathy D, Shete S, et al. UPAR and HER-2 gene status in individual breast cancer cells from blood and tissues. Proc Natl Acad Sci U S A. 2006;103(46):17361-17365.

16. Munzone E, Nole F, Goldhirsch A, et al. Changes of HER2 status in circulating tumor cells compared with the primary tumor during treatment for advanced breast cancer. Clin Breast Cancer. 2010;10(5):392-397.

17. Bidard F-C, Peeters DJ, Fehm T, et al. Clinical validity of circulating tumour cells in patients with metastatic breast cancer: a pooled analysis of individual patient data. Lancet Oncol. 2014;15(4):406-414.

18. Bidard FC, Mathiot C, Delaloge S, et al. Single circulating tumor cell detection and overall survival in nonmetastatic breast cancer. Ann Oncol. 2010;21(4):729-733.

19. Giordano A, Giuliano M, De Laurentiis M, et al. Circulating tumor cells in immunohistochemical subtypes of metastatic breast cancer: lack of prediction in HER2-positive disease treated with targeted therapy. Ann Oncol. 2012;23(5):1144-1150.

20. Liu Y, Liu Q, Wang T, et al. Circulating tumor cells in HER2-positive metastatic breast cancer patients: a valuable prognostic and predictive biomarker. BMC Cancer. 2013;13:202.

21. Allard WJ, Matera J, Miller MC, et al. Tumor cells circulate in the peripheral blood of all major carcinomas but not in healthy subjects or patients with nonmalignant diseases. Clin Cancer Res. 2004;10(20):6897-6904.

22. Frithiof H, Welinder C, Larsson AM, Ryden L, Aaltonen K. A novel method for downstream characterization of breast cancer circulating tumor cells following CellSearch isolation. J Transl Med. 2015;13(1):126.

23. Ross JS, Fletcher JA, Bloom KJ, et al. Targeted therapy in breast cancer: the HER-2/neu gene and protein. Mol Cell Proteomics. 2004;3(4): 379-398.

24. Cristofanilli M, Budd GT, Ellis MJ, et al. Circulating tumor cells, disease progression, and survival in metastatic breast cancer. $N$ Engl J Med. 2004;351(8):781-791.

25. Wolff AC, Hammond ME, Hicks DG, et al. Recommendations for human epidermal growth factor receptor 2 testing in breast cancer: American Society of Clinical Oncology/College of American Pathologists clinical practice guideline update. J Clin Oncol. 2013;31(31):3997-4013.

26. Kallioniemi OP, Kallioniemi A, Kurisu W, et al. ERBB2 amplification in breast cancer analyzed by fluorescence in situ hybridization. Proc Natl Acad Sci U S A. 1992;89(12):5321-5325.

27. Kuukasjarvi T, Karhu R, Tanner M, et al. Genetic heterogeneity and clonal evolution underlying development of asynchronous metastasis in human breast cancer. Cancer Res. 1997;57(8):1597-1604.

28. Prat A, Perou CM. Mammary development meets cancer genomics. Nat Med. 2009;15(8):842-844.

29. Neubauer H, Gall C, Vogel U, et al. Changes in tumour biological markers during primary systemic chemotherapy (PST). Anticancer Res. 2008;28(3B):1797-1804.

30. Pusztai L, Viale G, Kelly CM, Hudis CA. Estrogen and HER-2 receptor discordance between primary breast cancer and metastasis. Oncologist. 2010;15(11):1164-1168.

31. Pestrin M, Bessi S, Galardi F, et al. Correlation of HER2 status between primary tumors and corresponding circulating tumor cells in advanced breast cancer patients. Breast Cancer Res Treat. 2009;118(3): 523-530.

32. Paoletti C, Muniz MC, Thomas DG, et al. Development of circulating tumor cell-endocrine therapy index in patients with hormone receptor positive breast cancer. Clin Cancer Res. 2015;21(11):2487-2498. 
33. Ignatiadis $\mathrm{M}$, Rothe $\mathrm{F}$, Chaboteaux $\mathrm{C}$, et al. HER2-positive circulating tumor cells in breast cancer. PLoS One. 2011;6(1):e15624.

34. Swennenhuis JF, Tibbe AG, Levink R, Sipkema RC, Terstappen LW. Characterization of circulating tumor cells by fluorescence in situ hybridization. Cytometry A. 2009;75(6):520-527.

35. Bidard FC, Fehm T, Ignatiadis M, et al. Clinical application of circulating tumor cells in breast cancer: overview of the current interventional trials. Cancer Metastasis Rev. 2013;32(1-2):179-188.
36. Paik S, Kim C, Wolmark N. HER2 status and benefit from adjuvant trastuzumab in breast cancer. $N$ Engl J Med. 2008;358(13): 1409-1411.

37. Georgoulias V, Bozionelou V, Agelaki S, et al. Trastuzumab decreases the incidence of clinical relapses in patients with early breast cancer presenting chemotherapy-resistant CK-19mRNA-positive circulating tumor cells: results of a randomized phase II study. Ann Oncol. 2012;23(7): 1744-1750.

\section{Publish your work in this journal}

OncoTargets and Therapy is an international, peer-reviewed, open access journal focusing on the pathological basis of all cancers, potential targets for therapy and treatment protocols employed to improve the management of cancer patients. The journal also focuses on the impact of management programs and new therapeutic agents and protocols on

\section{Dovepress}

patient perspectives such as quality of life, adherence and satisfaction. The manuscript management system is completely online and includes a very quick and fair peer-review system, which is all easy to use. Visit http://www.dovepress.com/testimonials.php to read real quotes from published authors.

Submit your manuscript here: http://www.dovepress.com/oncotargets-and-therapy-journal 